\title{
Long noncoding RNA LINC00313 modulates papillary thyroid cancer tumorigenesis via sponging miR-4429
}

\author{
W. J. WU, H. YIN, J. J. HU, X. Z. WEI* \\ Department of Ultrasound, The Second Hospital of Ningbo, Ningbo City, China \\ ${ }^{*}$ Correspondence: $w x z 02130830 @ 163 . c o m$
}

Received February 19, 2018 / Accepted April 21, 2018

\begin{abstract}
Mounting evidence indicates that long noncoding RNAs (lncRNAs) play a critical role in tumorigenesis. LncRNA LINC00313 has been found to be up-regulated and associated with poor prognosis in lung cancer. However, the potential role and clinical value of LINC00313 in human papillary thyroid cancer (PTC) remain elusive and therefore require examination. The aim of this study is to investigate the role of LINC00313 in papillary thyroid cancer (PTC). We found its expression was significantly up-regulated in PTC tissues and cell lines and that this up-regulation correlated with poor prognosis. In vitro experiments indicated that down-regulation of LINC00313 inhibited proliferation and the migratory and colony-forming abilities of PTC cells. Moreover, silencing LINC00313 induced cell cycle arrest and apoptosis in the PTC cells. In addition, mechanism studies showed that LINC00313 down-regulates miR-4429 expression, and that miR-4429 over-expression can abrogate the oncogenic role of LINC00313 in PTC cells. In summary, our data revealed that LINC00313 acts as an oncogene in PTC via sponging miR-4429, and this suggests that LINC00313 may be successfully applied as a therapeutic target in PTC.
\end{abstract}

Key words: LINC00313, papillary thyroid cancer, miR-4429, cell cycle arrest, apoptosis

Thyroid carcinoma (TC) is the most common type of endocrine cancer, and its incidence rate has been increasing over the past decade [1]. It is estimated that there will be over sixty thousand new cases of TC, accounting for approximately $3.8 \%$ of all newly diagnosed malignancies, and that approximately two thousand TC patients will have died from this disease in the U.S. in 2016 [2]. There are three subtypes of TC: differentiated follicular carcinomas (FTC), papillary carcinomas (PTC), and de-differentiated and highly aggressive anaplastic carcinomas (ATC) [3].

However, PTC is the main form and accounts for over $80 \%$ of all TC cases [4]. Although PTC is normally an indolent cancer with favorable prognosis and low mortality rate, patients with advanced PTC still have only 59\% five-year survival rate [5]. It is therefore essential to identify novel biomarkers to improve diagnosis and develop novel therapeutic targets.

Long noncoding RNAs (lncRNA) are a group of RNA molecules with over 200 nucleotides and limited proteincoding potential [6]. Mounting evidence indicates that multiple lncRNAs have important roles in various pathophysiological processes and that the expression of lncRNAs is often deregulated in many diseases, including TC. For example, IncRNA SPRY4-IT was significantly up-regulated in TC tissues and associated with poor prognosis [7]. Further, low expression of $\operatorname{lncRNA}$ CASC2 promotes tumorigenesis and indicates poor prognosis for TC patients [8], as is also increased lncRNA H19 expression [9]. LINC00313 is a novel identified lncRNA found to be up-regulated and function as an oncogene in lung cancer [10]. Moreover, LINC00313 is up-regulated in fibrolamellar carcinoma (FLC) which is a unique liver cancer primarily affecting young adults [11]. While these findings indicate that LINC00313 has an essential role in the development of various cancers, its actual function in PTC remains elusive.

Herein, we investigated the biological functions of LINC00313 in PTC cells, and results reveal that it is up-regulated in PTC tissues and cell lines. Moreover, further investigations indicated that down-regulation of LINC00313 inhibits proliferation, invasion and colony formation in PTC cells and its down-regulation induces apoptosis and cell cycle arrest in the G2/M phase. Furthermore, LINC00313 was identified as a competing endogenous RNA for miR-4429, and these findings now provide a novel therapeutic target for PTC. 


\section{Patients and methods}

Patients and tissues. A total of 26 confirmed pathologically papillary thyroid cancer tissues (PTC) and paired adjacent normal thyroid tissues, were obtained from PTC patients who underwent radical surgical resection at the Second Hospital of Ningbo City. No patients received radiotherapy or chemotherapy before surgery. Collected tissues were immediately frozen in liquid nitrogen and stored at $-80^{\circ} \mathrm{C}$ for subsequent experiments. Informed consent was obtained from all patients and the Ethical Committee of the Second Hospital of Ningbo City for Clinical Research approved this study.

Cell culture. Human thyroid papillary cell lines (K1, IHH4, MDA-T32, NPA87, TPC-1) and one normal thyroid cell line (Nthy-ori 3-1) authenticated and tested for mycoplasma contamination were purchased from BeNa Culture Collection (Beijing, China). All cells were cultured in Dulbecco's Modified Eagle's Medium (DMEM) (Life Technologies, Carlsbad, CA, USA) supplemented with $10 \%$ fetal bovine serum (FBS) (Gibco), $100 \mathrm{U} / \mathrm{ml}$ penicillin and $100 \mu \mathrm{g} / \mathrm{ml}$ streptomycin (Thermo Fisher, Waltham, MA, USA). Cells were maintained in an incubator with a humidified atmosphere containing $5 \% \mathrm{CO}_{2}$ at $37^{\circ} \mathrm{C}$.

Quantitative real-time PCR. Total RNA was extracted from frozen tissues and cells with TRIzol reagent (Life Technologies) according to the manufacturer's guide. The quantity of RNA was measured by a NanoDrop 1000 spectrophotometer (Thermo Fisher, Wilmington, DE, USA). The ratio of the absorbance at 260 and $280 \mathrm{~nm}$ (A260/A280 $\geq 1.8$ ) assessed the quality of RNA. Quantitative real-time PCR was carried out using SYBR Premix Ex $\mathrm{Taq}^{\mathrm{TM}}$ (Takara, Dalian, China) according to the manufacturer's guide for the StepOnePlus ${ }^{\mathrm{TM}}$ real-time PCR system (Applied Biosystems, Foster City, CA, USA). The following primers were used: LINC00313: Forward: 5'-TTGCGTGACAGTTTCCACTC-3'; Reverse: 5'-CTCCCTTCTGCGGTCATTTC-3'; miR-4429: Forward: 5'-ATTATTGGGGCTGGGCG-3'; Reverse: 5'-CAGTGCAGGGTCCGAGGT-3'; U6: Forward: 5'-TGCGGGTGCTCGCTTCGGCAGC-3'; Reverse: 5'-CCAGTGCAGGGTCCGAGGT-3'; GAPDH: Forward: 5'-CTGGGCTACACTGAGCACC-3'; Reverse: 5'-AGTGGTCGTTGAGGGCAATG-3'.

The levels of LINC00313 and miR-4429 were calculated by $2^{-\Delta \Delta \mathrm{Ct}}$ method and normalized to the level of U6 and GAPDH, respectively. All assays were performed in triplicate. The expression levels were expressed as the fold change relative to the corresponding controls, which were defined as 1.0 .

Cell transfection. Specific siRNA against LINC00313 (si-LINC00313), negative control siRNA (si-NC), miR-4429 mimic, and negative control mimic (miR-NC) were designed and synthesized by GenePharm Company (Suzhou, China). Transfection was performed with Lipofectamine 2000 (Invitrogen) according to the manufacturer's guide.
Cell viability assay. Cell viability was assayed as previously described [12]. Briefly, $5 \times 10^{3}$ cells/well were seeded in a 96-well plate. $20 \mathrm{~mL} 3-(4,5)$-dimethylthiahiazo(-z-y1)3,5-diphenytetrazoliumromide (MTT) solution $(5 \mathrm{mg} / \mathrm{ml})$ (Sigma, St Louis, USA) was added to each well. Four hours after incubation, $150 \mu \mathrm{l}$ of dimethyl sulfoxide (DMSO) was added to each well to terminate the reaction. Absorbance was then measured at $450 \mathrm{~nm}$ by spectrophotometer (BioTek, Winooski, VT, USA). Three independent experiments were performed.

Apoptosis assay. Apoptosis was measured by Cell Death Detection ELISA kit (Roche, Mannheim, Germany) according to the manufacturer's guide. Briefly, cells were seeded in 96-well plates at a density of $5 \times 10^{3}$ cells/well. After transfection for $24 \mathrm{~h}$, the cells were lysed and lysates were transferred into 96-well plates coated with anti-histone antibodies. Peroxidase-conjugated anti-DNA antibody was added after 2-hours incubation. Finally, after adding ABTS substrate, the absorbance was measured at $405 \mathrm{~nm}$ by spectrophotometer (BioTek). Three independent experiments were performed.

Caspase-3 activity assay. The activity of caspase- 3 was measured by caspase- 3 activity kit (Beyotime Biotechnology, Shanghai, China) according to the manufacturer's guide. After transfection for $24 \mathrm{~h}$, cells were collected and lysed in the provided buffer with caspase- 3 substrate Ac-DEVDpNA $(2 \mathrm{mM})$. Lysates were then incubated at $37^{\circ} \mathrm{C}$ for $2 \mathrm{~h}$. The absorbance of the samples was measured at $40 \mathrm{~nm}$ by spectrophotometer (BioTek). Three independent experiments were performed.

Cell cycle analysis. Cells were seeded in a 96-well plate at a density of $5 \times 10^{3}$ cells/well. After transfection for $24 \mathrm{~h}$, cells were collected and fixed in $70 \%$ ethanol. Then, the cells were washed with PBS and stained with PI (Sigma) in the dark at room temperature for $30 \mathrm{~min}$. The distribution of the cell cycle phase was then analyzed by flow cytometry (BD Biosciences, San Jose, CA, USA). The percentages of cells at different phases of the cell cycle were calculated from three independent experiments.

Transwell experiments. We used 24-well Transwell ${ }^{\mathrm{TM}}$ plates (Corning, NY, USA) to assay migration ability. After transfection, $2 \times 10^{5}$ cells were seeded in the top chamber which was supplemented with $200 \mu$ l of medium without serum. The bottom well contained medium with 20\% FBS. After $24 \mathrm{~h}$, the cells in the upper chamber were fixed with methanol and stained with crystal violet. Migratory cells were photographed by Nikon Eclipse E600 microscope (Nikon Instruments). All experiments were carried out three times independently.

Luciferase reporter assay. The pmirGLO, pmirGLOLINC00313 wt or pmirGLO-LINC00313 mut was co-transfected with miR-4429 mimics or miR-NC mimics into cells by Lipofectamine 2000 (Life Technologies) according to the manufacturer's guide. Forty-eight hours after transfection, cells were harvested and analyzed for luciferase activity using 
a Dual-Glo Luciferase Assay System (Promega, WI, USA). Relative luciferase activity was calculated as the ratio of raw firefly luciferase activity to renilla luciferase activity.

Colony formation assay. Transfected cells were seeded in a 6 -well plate at a density of $5 \times 10^{3}$ cells/well and replenished every 3 days with fresh medium. Cells were fixed with methanol and stained with crystal violet 2 weeks later. Visible colonies were manually counted in randomly selected fields with a Nikon Eclipse E600 microscope (Nikon Instruments). The clone formation rate (CFR) was calculated according to the following formula: $\mathrm{CFR}=$ clone counts/seeded cell counts $\times 100 \%$. All experiments were carried out three times independently.

Western blot analysis. Transfected cells were collected and lysed with RIPA lysis buffer (Beyotime). Equal amounts of protein extractions $(20 \mu \mathrm{g})$ were subjected to SDS-PAGE and transferred to PVDF membranes which were then blocked with $5 \%$ skimmed milk for $2 \mathrm{~h}$ at room temperature. The membranes were incubated at $4{ }^{\circ} \mathrm{C}$ overnight with the following primary antibodies: Cyclin D1, Cdc25A (Abcam, Cambridge, MA, USA), p21, Bcl-2, Mcl-1, Bax, Caspase-3 (CST, Danvers, MA, USA) and GAPDH (Sigma). Membranes were then incubated with HRP-labelled secondary antibodies at room temperature for $1 \mathrm{~h}$. The signals were detected using an enhanced chemiluminescence-detecting kit (Thermo Fisher), followed by analysis by Tanon 5200 Chemiluminescent Imaging System (Tanon, Shanghai, China). Proteins were quantified using GAPDH as a loading control.

Statistical analysis. Data is presented as mean \pm standard error of at least three independent experiments. The SPSS 17.0 software (SPSS Inc., Chicago, USA) provided statistical analysis. Two-group comparisons were performed using the Student's t-test and multiple-group comparisons were analyzed by one-way ANOVA. The correlation between the LINC00313 and miR-4429 expression levels in 26 cases of PTC tissues was analyzed by Spearman's correlation. A p-value $<0.05$ was considered statistically significant.

\section{Results}

LINC00313 is increased in PTC tissues and cell lines. To investigate the expression pattern and clinical significances of lncRNA LINC00313 in PTC, we first measured the expression of LINC00313 in 26 PTC tissues and paired adjacent normal thyroid tissues. As indicated in Figure 1A, LINC00313 was significantly up-regulated in PTC tissues compared with normal tissues $(\mathrm{p}<0.01)$. We then investigated the diagnostic values of LINC00313 for PTC. ROC analysis revealed that LINC00313 could efficiently discriminate PTC tissues from normal tissues (Figure 1B). The area under the curve (AUC) was 0.8166 (95\% CI: 0.7035-0.9296, p<0.001), thus suggesting that LINC00313 expression can be applied as a biomarker for PTC. Furthermore, our results showed that LINC00313 expression was significantly increased in PTC cell lines (K1, IHH-4, MDA-T32, NIPA87 and TPC-1) compared with a normal thyroid follicular epithelial cell line (Nthy-ori 3-1), and the expression of LINC00313 in PTC cell lines was higher than that in Nthy-ori 3-1 (Figure 1C). We then analyzed the relationship between the expression of LINC00313 and clinical-pathological characteristics of these 26 PTC tissue pairs. Table 1 shows that the high expression of LINC00313 was associated with tumor stage (T3-T4) $(\mathrm{p}=0.002)$, lymph node metastasis $(\mathrm{p}=0.018)$ and TNM stage (III-IV) $(\mathrm{p}=0.005)$. This implies that LINC00313 may be involved in the tumorigenesis of PTC.

Silencing of LINC00313 impairs proliferation of PTC cells. To explore the biological function of LINC00313 in PTC cells, we used specific small interfering RNA to knockdown LINC00313 in two PTC cell lines, TPC1 and SW579. The transfection efficiency was evaluated at $48 \mathrm{~h}$ post-trans-
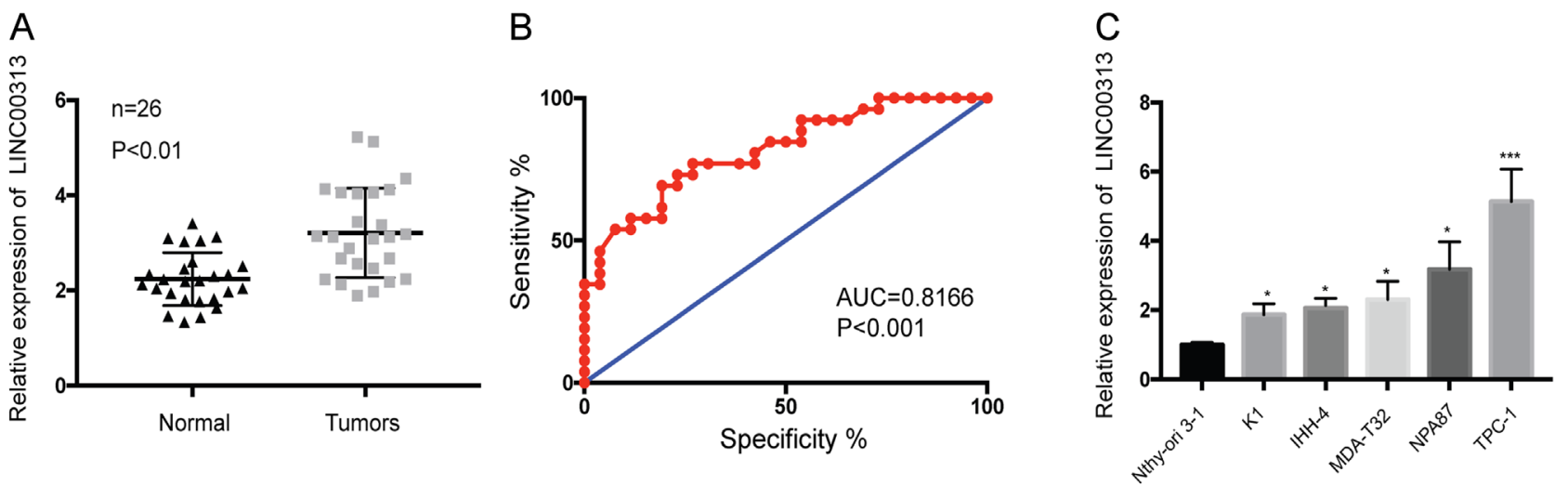

Figure 1. The expression of LINC00313 was down-regulated in PTC tissues and cell lines. A) LINC00313 expression was determined by qRT-PCR in 26 PTC tissues and paired adjacent normal tissues. B) ROC curve for LINC00313 based on the qRT-PCR data. The diagram is a plot of the sensitivity (Y-axis, true-positive rate) vs. specificity (X-axis, false-positive rate) over all possible CT values. The AUC values indicate that the two groups may be distinguished by expression analysis of the markers. AUC: 0.8166, (95\% CI: 0.7035-0.9296). ROC: receiver operating characteristic; AUC: area under the curve. C) LINC00313 expression levels in PTC cells and normal thyroid follicular epithelial cell line (Nthy-ori 3-1) were determined by qRT-PCR. Data is by mean $\pm \mathrm{SD},{ }^{*} \mathrm{p}<0.05 ;{ }^{* * *} \mathrm{p}<0.001$. Each experiment was repeated three times. 
Table 1. Association between LINC00313 expression and clinical-pathological characteristics of the PTC patients.

\begin{tabular}{|c|c|c|c|}
\hline \multirow{2}{*}{ Parameters } & \multicolumn{2}{|c|}{ Expression of LINC00313 } & \multirow{2}{*}{ p-value } \\
\hline & Low & High & \\
\hline Gender & & & 0.691 \\
\hline Male & 5 & 6 & \\
\hline Female & 8 & 7 & \\
\hline Age (years) & & & 0.680 \\
\hline$<45$ & 4 & 5 & \\
\hline$\geq 45$ & 9 & 8 & \\
\hline Focality & & & 0.420 \\
\hline Unifocal & 7 & 9 & \\
\hline Multifocal & 6 & 4 & \\
\hline Extrathyroidal extension & & & 0.658 \\
\hline Positive & 3 & 4 & \\
\hline Negative & 10 & 9 & \\
\hline Primary tumor & & & 0.002 \\
\hline $\mathrm{T} 1-\mathrm{T} 2$ & 10 & 2 & \\
\hline $\mathrm{T} 3-\mathrm{T} 4$ & 3 & 11 & \\
\hline Lymph node metastasis & & & 0.018 \\
\hline No & 9 & 3 & \\
\hline N1 & 4 & 10 & \\
\hline TNM stage & & & 0.005 \\
\hline I-II & 9 & 2 & \\
\hline III-IV & 4 & 11 & \\
\hline
\end{tabular}

A

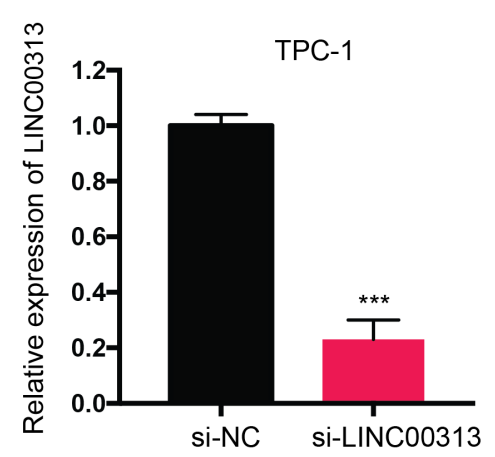

B

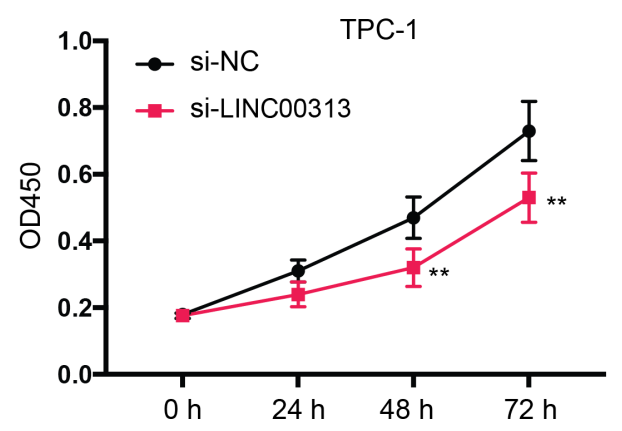

fection (Figure 2). The MTT assay results showed diminished proliferation ability in si-LINC00313-transfected cells compared with that in si-NC-transfected cells.

Down-regulation of LINC00313 represses colony formation, migration and cell cycle progression in PTC cells. We examined the effects of LINC00313 on colony formation ability in PTC cells. As shown in Figure 3A, down-regulation of LINC00313 significantly decreased colony formation ability in PTC cells. Additionally, the transwell assay showed that silencing LINC00313 c weakens the migration capacity of PTC cells (Figure 3B). We further detected cell cycle distribution of the transduced TPC1 and NPA87 cells using flow cytometry. The results indicated significant decrease in the $S$ phase and increase in the G2/M phase in the numbers of both cell lines transduced with si-LINC00313 compared to control cells. This suggests that LINC00313 modulates cell cycle progression in PTC cells (Figure 3C). In addition, the protein expression levels of cell-cycle progression-related proteins were measured by western blotting. The results showed that the expression levels of Cyclin D1 and Cdc25A were decreased and that p21 expression was increased by LINC00313 knockdown (Figure 3D. The combined data demonstrates that LINC00313 is involved in the progression of PTC cells.
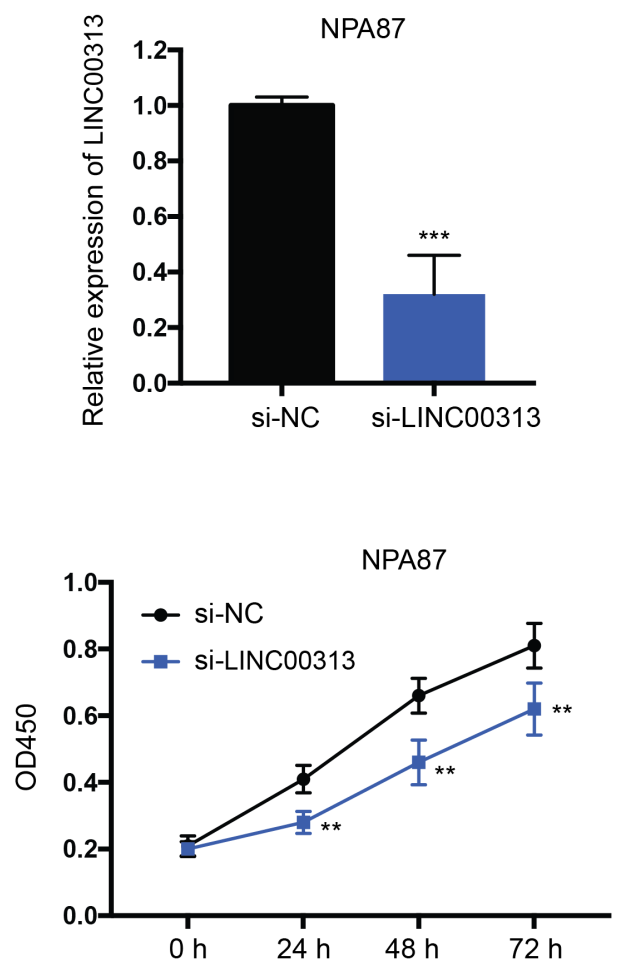

Figure 2. Down-regulation of LINC00313 inhibits proliferation of PTC cells. A) TPC-1 and NPA87 cells were transfected with either si-LINC00313 or si-NC for $24 \mathrm{~h}$, and the levels of LINC00313 were then measured by qRT-PCR. B) TPC-1 and NPA87 cells were transfected with si-LINC00313 or si-NC for the indicated time and cell viabilities were then MTT assayed. Data is by mean $\pm \mathrm{SD},{ }^{\star *} \mathrm{p}<0.01 ;{ }^{\star * *} \mathrm{p}<0.001$. Each experiment was repeated three times. 

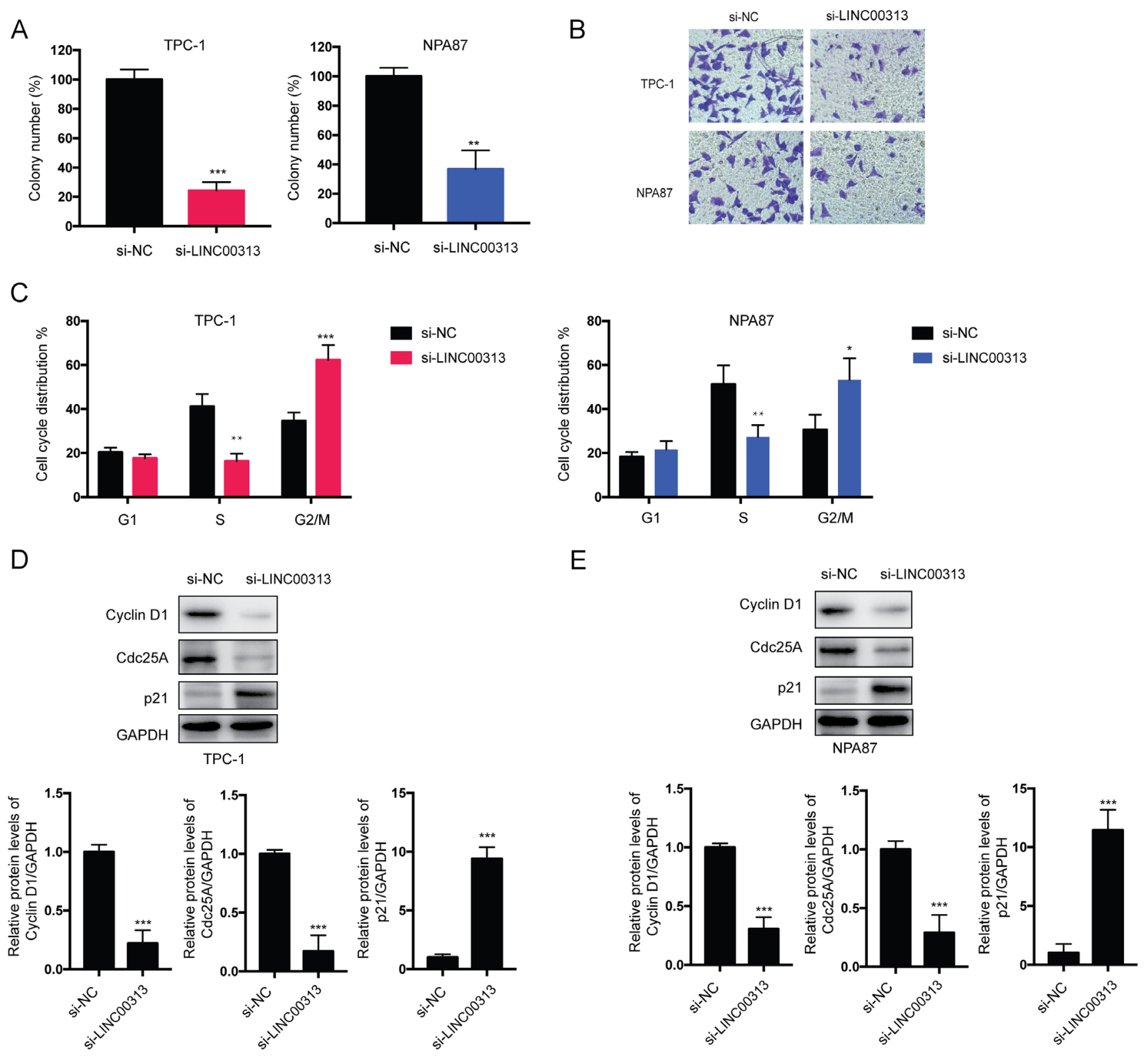

Figure 3. LINC00313 affects colony formation, migration and cell cycle progression in TPC cells. A) Colony formation of TPC-1 and NPA87 cells treated as indicated. The colonies were counted on day 7 following transfection. B) Migration assay of TPC-1 and NPA87 cells treated as indicated. After $24 \mathrm{~h}$, the number of cells migrated through the membrane was counted under a microscope using five random fields (magnification, $\times 100$ ). $\mathrm{C}$ ) Cell cycle distribution analysis of TPC-1 and NPA87 cells treated as indicated. Knockdown of LINC00313 resulted in more cells arrested in the G2/M phase. D) TPC-1 and NPA87 cells were treated as indicated for $24 \mathrm{~h}$, and total cellular lysates were then collected and subjected to western blot analysis with the indicated antibodies. The western blot results were quantitatively analyzed. Data is by mean $\pm \mathrm{SD},{ }^{\star} \mathrm{p}<0.05 ;{ }^{\star *} \mathrm{p}<0.01 ;{ }^{\star * *} \mathrm{p}<0.001$. Each experiment was repeated three times.

Knockdown of LINC00313 promoted apoptosis and modulated the expression of Bcl-2 proteins in PTC cells. We investigated if LINC00313 affected apoptosis in PTC cells. The apoptosis assay revealed that relative apoptotic cells were increased in the si-LINC00313 group compared to those in the control group ( $\mathrm{p}<0.001)$ (Figure $4 \mathrm{~A})$. The caspase- 3 activity assay also confirmed significant increase in caspase-3 activity after transfection with si-LINC00313 compared to the control group (Figure 4B). The expression levels of apoptosis-related factors were also measured by western blotting. As shown in Figure 4C, the expression levels of antiapoptotic Bcl-2 and Mcl-1 were decreased while the level of pro-apoptotic Bax was increased after transfection with si-LINC00313 compared to si-NC. The Bax/Bcl-2 ratio was then quantified and an increase in $\mathrm{Bax} / \mathrm{Bcl}-2$ ratio was observed in the si-LINC00313 group compared to the 

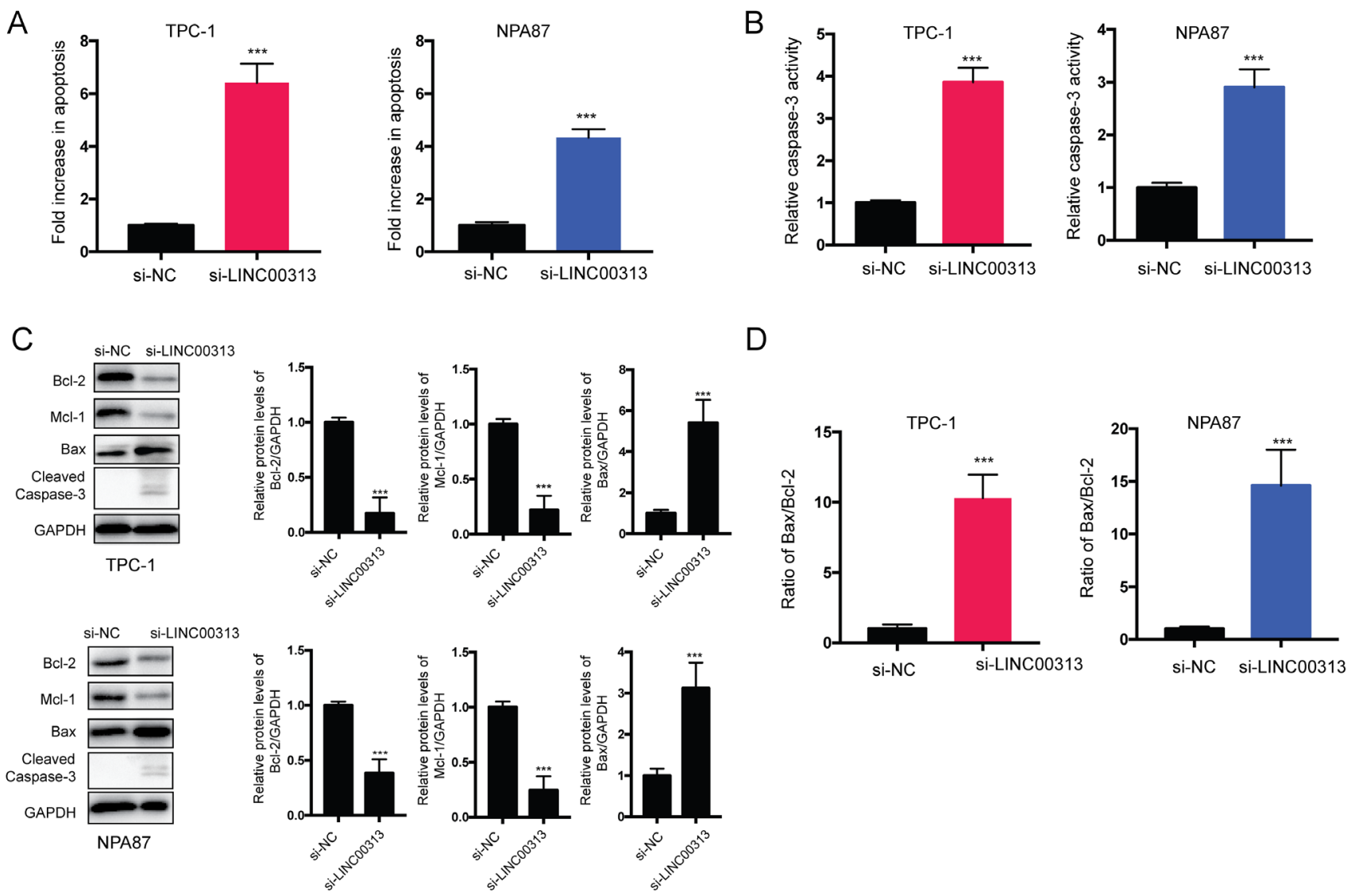

Figure 4. LINC00313 affects apoptosis and the levels of Bcl-2 proteins. A) TPC-1 and NPA87 cells were treated as indicated for $24 \mathrm{~h}$ and the relative cellular apoptosis rate was determined by ELISA assay. B) TPC-1 and NPA87 cells were treated as indicated and the relative caspase-3 activity was assayed. C) TPC-1 and NPA87 cells were treated as indicated, and total cellular lysates were collected and subjected to western blot analysis. The western blot results were quantitatively analyzed. D) The relative ratio of Bax/Bcl-2 was quantified. Data is by mean $\pm \mathrm{SD}$, ${ }^{* * *}$ p $<0.001$. Each experiment was repeated three times.

control group (Figure 4D). This established that downregulation of LINC00313 leads to apoptosis of PTC cells via modulation of $\mathrm{Bcl}-2$ proteins.

LINC00313 sponges and downregulates miR-4429. Various lncRNAs have recently been identified as competing endogenous RNAs (ceRNA) via competitively binding miRNAs $[13,14]$. To examine whether LINC00313 exerts its function as a ceRNA in PTC, we utilized bioinformatics tools (TargetScan and miRanda) and identified miR-4429 as a putative binding miRNA. We then examined the expression levels of miR-4429 in PTC tissues and adjacent normal tissues. The results indicated that miR-4429 expression was significantly down-regulated in PTC tissues compared to corresponding adjacent normal tissues $(\mathrm{p}<0.01$, Figure $5 \mathrm{~A})$. The ROC curve of miR- 4429 predicted the prognosis of PTC and results indicated that the area under the curve (AUC) of miR-4429 was 0.7589 ( $\mathrm{p}<0.001$, Figure $5 \mathrm{~B})$, suggesting that miR-4429 expression may also serve as a biomarker for PTC. Moreover, we found negative correlation between LINC00313 and miR-4429 expression levels in PTC tissues $(\mathrm{p}<0.05)$. To further confirm the relationship between LINC00313 and miR-4429, a luciferase reporter assay was performed. As shown in Figure 5E, miR-4429 mimics reduced the luciferase activity of wild-type LINC00313 reporter vector (wt) but not that of the mutant reporter vector (mut) in both PTC cell lines.

Effects of miR-4429 over-expression on proliferation, apoptosis and cell cycle progression in PTC cells. To further investigate the functional roles of miR-4429 in PTC, a series of in vitro assays were performed. The overexpression of miR-4429 was successfully achieved by transfecting both PTC cell lines with miR-4429 mimics (Figure 6A). MTT assays showed that the over-expression of miR-4429 significantly suppressed cell proliferation of PTC cells (Figure 6B). The up-regulation of miR-4429 also promoted apoptosis of PTC cells (Figure 6C). Moreover, over-expression of miR-4429 induced cell cycle arrest in the G2/M phase in PTC cells (Figure 6D). This suggests that LINC00313 exert its function, at least partly, by negative regulation of miR-4429. 
A

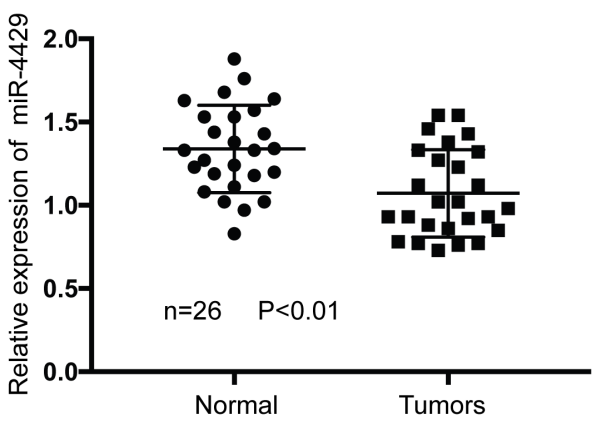

C

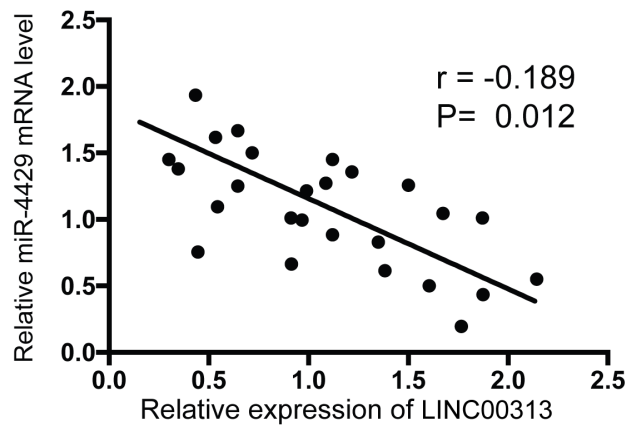

$E$

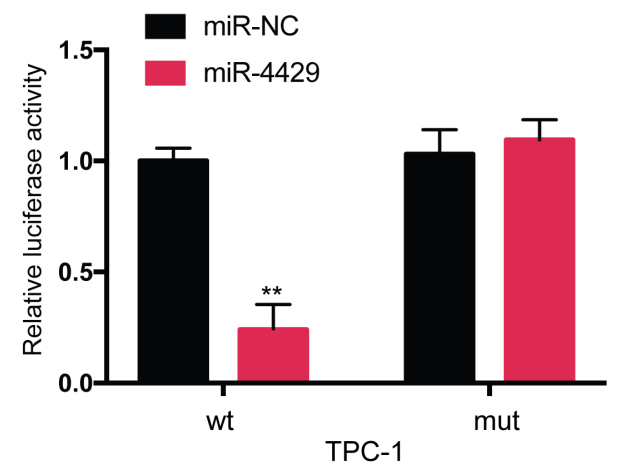

B

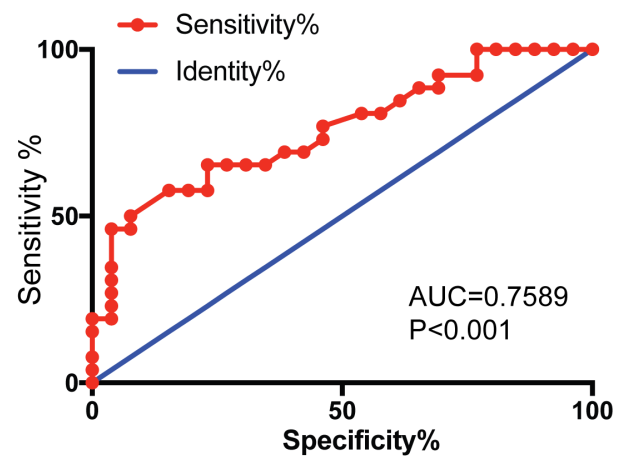

D

Figure 5. LINC00313 sponges miR-4429. A) miR-4429 expression levels in 26 PTC tissues and paired adjacent normal tissues were determined by qRTPCR. B) Diagnostic values of miR-4429 for discriminating PTC tissues from normal tissues. AUC: 0.7589, (95\% CI: 0.6287-0.8891). C) The expression of LINC00313 was inversely related to miR-4429 in PTC tissues. D) The putative binding sites between LINC00313 and miR-4429. E) Luciferase assays were performed to determine the association between LINC00313 and miR-4429 in PTC cells. Data is presented as the relative ratio of firefly luciferase activity to renilla luciferase activity. Data is by mean $\pm S D,{ }^{\star *} p<0.01$. Each experiment was repeated three times.

\section{Discussion}

Mounting evidence indicates that deregulation of lncRNA contributes to a variety of biological activities including tumorigenesis [15]. Many $\operatorname{lncRNAs}$ are related to PTC and their functions have recently been studied. For example, lncRNA CCND2-AS1 functions as an oncogene and promotes tumorigenesis in PTC [16]. LncRNA NEAT1 promoted carcinogenesis and undesirable progression of PTC via modulating miR-129-5p/KLK7 expression [17]. Other well-known lncRNAs, such as MALAT1 and H19, have shown oncogenic or tumor inhibitor functions during PTC development $[18,19]$. This evidence indicates that lncRNAs possess potential as markers for diagnosis and prognosis and 

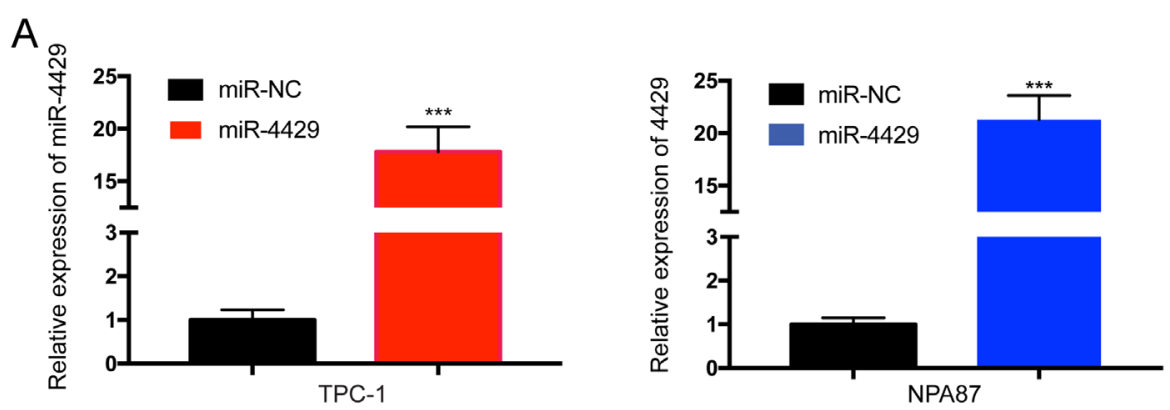

B
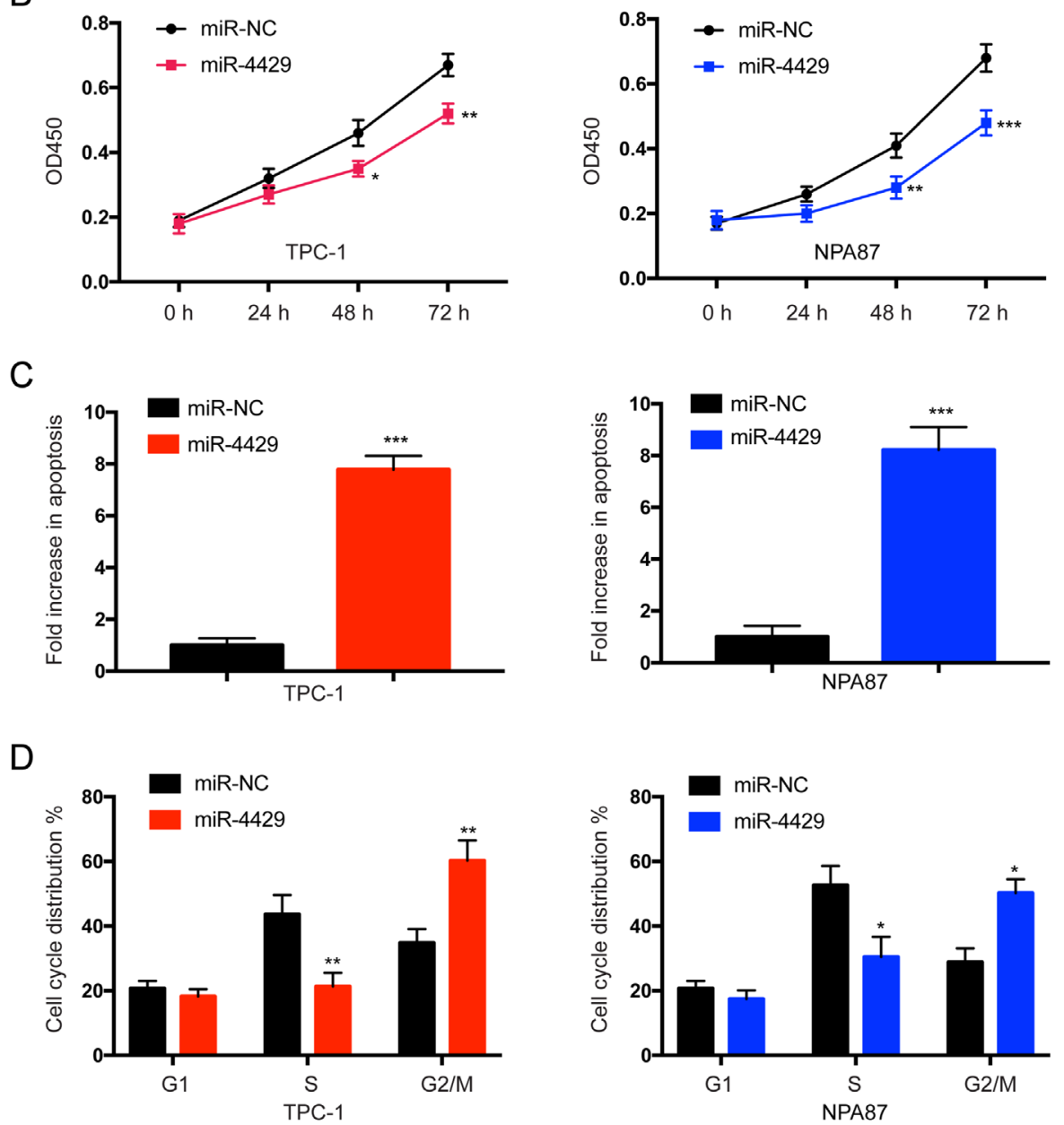

Figure 6. Effects of miR-4429 on viability, apoptosis and cell cycle progression in PTC cells. A) PTC cells were transfected with miR-NC or miR-4429 mimics and the levels of miR-4429 were assayed by QRT-PCR. B) TPC-1 and NPA87 cells were treated as indicated, and cell viabilities were MTT assayed. C) TPC-1 and NPA87 cells were treated as indicated for $24 \mathrm{~h}$ and the relative cellular apoptosis rate was measured by ELISA assay. D) TPC-1 and NPA87 cells were transfected with miR-NC or miR-4429 mimics for $24 \mathrm{~h}$ and the cell cycle distribution was assayed. Data is $\mathrm{b}$ mean $\pm \mathrm{SD},{ }^{*} \mathrm{p}<0.05$; ${ }^{* *} \mathrm{p}<0.01 ;{ }^{* * *} \mathrm{p}<0.001$. Each experiment was repeated three times.

possible therapy targets for PTC. Although many lncRNAs have been investigated, there is little information about the clinical significance and biological function of LINC00313 in PTC.

Herein, we demonstrated that LINC00313 is highly expressed in PTC tissues and cell lines. High expression levels of LINC00313 had strong association with lymph node metastasis and advanced tumor TNM stages in PTC patients. Furthermore, LINC00313 could efficiently discriminate PTC from non-cancerous tissue. Therefore, our results suggest that LINC00313 could be a potential biomarker for diagnosing PTC and predicting lymph node metastasis in 
PTC. Our findings also agree with a previous study where expression levels of LINC00313 correlated with shorter overall survival in lung cancer patients [10]. In vitro studies indicated that down-regulation of LINC00313 distinctly repressed proliferation, colony formation and migration in PTC cells. LINC00313 has also been found to regulate cell cycle progression and cell cycle-related proteins. Moreover, silencing LINC00313 leads to apoptosis of PTC cells accompanied by modulation of Bcl-2 family proteins. There are two well-characterized pathways that contribute to apoptosis extrinsic and intrinsic pathways [20]. The intrinsic pathway is strictly subjected to regulation of $\mathrm{Bcl}-2$ family proteins [21]. In our study, silencing LINC00313 led to down-regulation of anti-apoptotic Bcl-2 proteins Bcl-2 and Mcl-1 and the up-regulation of pro-apoptotic Bcl-2 protein Bax. Therefore, silencing LINC00313 can trigger apoptosis via the intrinsic pathway; but further investigation is needed.

A number of studies have demonstrated that lncRNAs are able to function as competitive endogenous RNAs (ceRNAs) which negatively regulate the expression of miRNAs [22]. In our study, bioinformatics analysis predicted that miR-4429 binds with LINC00313. The luciferase assay then revealed that LINC00313 directly binds miR-4429 and we found negative correlation between LINC00313 and miR-4429 expression levels in PTC tissues. Furthermore, over-expression of miR-4429 mimics the effects of silencing LINC00313 in PTC cells. Therefore, we conclude that LINC00313 promotes PTC development by negative regulation of miR-4429. Although further investigation is required, our data determined that miR-4429 may function as a tumor suppressor in PTC.

\section{Conclusion}

The experimental results demonstrated that LINC00313 is highly expressed in PTC tissues and correlates with poor clinical outcomes. Silenced LINC00313 repressed proliferation and migration ability in PTC cells and it also promoted their apoptosis and cell cycle arrest. Mechanism assays confirmed this effect is at least partly achieved by regulating miR-4429. Finally, the combined results provide a new perspective of LINC00313 function in papillary thyroid cancer tumorigenesis.

Acknowledgments: We thank Dr Jinwei Li for the technical assistance provided in this study.

\section{References}

[1] PELlEGRITI G, FRASCA F, REGALbUTO C, SQUATRITO S, VIGNERI R. Worldwide increasing incidence of thyroid cancer: update on epidemiology and risk factors. J Cancer Epidemiol 2013; 2013: 965212. https://doi. org/10.1155/2013/965212[2] SIEGEL RL, MILLER KD, JEMAL A. Cancer statistics, 2016. CA Cancer J Clin 2016; 66: 7-30. https://doi.org/10.3322/caac. 21332
[3] FLOOR SL, HEBRANT A, PITA JM, SAISELET M, TRESALLET $\mathrm{C}$ et al. MiRNA expression may account for chronic but not for acute regulation of mRNA expression in human thyroid tumor models. PLoS One 2014; 9: e111581. https://doi.org/10.1371/journal.pone.0111581

[4] GUILMETTE J, NOSE V. Hereditary and familial thyroid tumors. Histopathology 2018; 72: 70-81. https://doi. org $/ 10.1111 /$ his. 13373

[5] XING M. Molecular pathogenesis and mechanisms of thyroid cancer. Nat Rev Cancer 2013; 13: 184-199. https://doi. org/10.1038/nrc3431

[6] BATISTA PJ, CHANG HY. Long noncoding RNAs: cellular address codes in development and disease. Cell 2013; 152: 1298-1307. https://doi.org/10.1016/j.cell.2013.02.012

[7] ZHOU H, SUN Z, LI S, WANG X, ZHOU X. LncRNA SPRY4-IT was concerned with the poor prognosis and contributed to the progression of thyroid cancer. Cancer Gene Ther 2018; 25: 39-46. https://doi.org/10.1038/s41417-0170003-0

[8] XIONG X, ZHU H, CHEN X. Low expression of long noncoding RNA CASC2 indicates a poor prognosis and promotes tumorigenesis in thyroid carcinoma. Biomed Pharmacother 2017; 93: 391-397. https://doi.org/10.1016/j. biopha.2017.06.063

[9] LIU N, ZHOU Q, QI YH, WANG H, YANG L et al. Effects of long non-coding RNA H19 and microRNA let7a expression on thyroid cancer prognosis. Exp Mol Pathol 2017; 103: 71-77. https://doi.org/10.1016/j.yexmp.2017.06.004

[10] LI M, QIU M, XU Y, MAO Q, WANG J et al. Differentially expressed protein-coding genes and long noncoding RNA in early-stage lung cancer. Tumor Biol 2015; 36: 9969-9978. https://doi.org/10.1007/s13277-015-3714-6

[11] DINH TA, VITUCCI EC, WAUTHIER E, GRAHAM RP, PITMAN WA et al. Comprehensive analysis of The Cancer Genome Atlas reveals a unique gene and non-coding RNA signature of fibrolamellar carcinoma. Sci Rep 2017; 7: 44653. https://doi.org/10.1038/srep44653

[12] YU R, YU BX, CHEN JF, LV XY, YAN ZJ et al. Anti-tumor effects of Atractylenolide I on bladder cancer cells. J Exp Clin Cancer Res 2016; 35: 40. https://doi.org/10.1186/s13046016-0312-4

[13] DENG L, YANG SB, XU FF, ZHANG JH. Long noncoding RNA CCAT1 promotes hepatocellular carcinoma progression by functioning as let-7 sponge. J Exp Clin Cancer Res 2015; 34: 18. https://doi.org/10.1186/s13046-015-0136-7

[14] SALMENA L, POLISENO L, TAY Y, KATS L, PANDOLFI PP. A ceRNA hypothesis: the Rosetta Stone of a hidden RNA language? Cell 2011; 146: 353-358. https://doi.org/10.1016/j. cell.2011.07.014

[15] SPIZZO R, ALMEIDA MI, COLOMBATTI A, CALIN GA. Long non-coding RNAs and cancer: a new frontier of translational research? Oncogene 2012; 31: 4577-4587. https:// doi.org/10.1038/onc.2011.621

[16] XIA E, BHANDARI A, SHEN Y, ZHOU X, SINDAN N et al. LncRNA CCND2-AS1 promotes proliferation, migration, and invasion in papillary thyroid carcinoma. Biochem Biophys Res Commun 2018; 496: 628-632. https://doi. org/10.1016/j.bbrc.2018.01.102 
[17] ZHANG H, CAI Y, ZHENG L, ZHANG Z, LIN X et al. Long noncoding RNA NEAT1 regulate papillary thyroid cancer progression by modulating miR-129-5p /KLK7 expression. J Cell Physiol 2018. https://doi.org/10.1002/jcp.26425

[18] ZHANG R, HARDIN H, HUANG W, CHEN J, ASIOLI S et al. MALAT1 Long Non-coding RNA Expression in Thyroid Tissues: Analysis by In Situ Hybridization and Real-Time PCR. Endocr Pathol 2017; 28: 7-12. https://doi.org/10.1007/ s12022-016-9453-4

[19] LAN X, SUN W, DONG W, WANG Z, ZHANG T et al. Downregulation of long noncoding RNA H19 contributes to the proliferation and migration of papillary thyroid carcinoma. Gene 2018; 646: 98-105. https://doi.org/10.1016/j. gene.2017.12.051
[20] HENGARTNER MO. The biochemistry of apoptosis. Nature 2000; 407: 770-776. https://doi.org/10.1038/35037710

[21] SCHWARTZMAN RA, CIDLOWSKI JA. Apoptosis: the biochemistry and molecular biology of programmed cell death. Endocr Rev 1993; 14: 133-151. https://doi.org/10.1210/ edrv-14-2-133

[22] CESANA M, CACCHIARELLI D, LEGNINI I, SANTINI T, STHANDIER O et al. A long noncoding RNA controls muscle differentiation by functioning as a competing endogenous RNA. Cell 2011; 147: 358-369. https://doi.org/10.1016/j. cell.2011.09.028 